\title{
Análisis del desempeño laboral de los graduados universitarios desde su propia perspectiva. Estudios en Lengua y Literatura Hispánicas de la Facultad de Educación e Idiomas de la UNAN-Managua
}

Dra. Rocío Cruz-Díaz

Facultad de Ciencias Sociales

Universidad Pablo de Olavide, Sevilla

mrcrudia@upo.es

MSc. Nora Cuadra Baquedano

Facultad de Educación e Idiomas

UNAN-MANAGUA

norcedes@yahoo.com

Palabras clave: Investigación, Educación Superior, Seguimiento a graduados, Evaluación, Desempeño Laboral.

\section{RESUMEN}

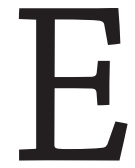

n este artículo, la autoría presenta las evidencias investigadoras interesadas en la evaluación institucional obtenidas de una sección del estudio titulado: Desempeño y satisfacción laboral de los graduados de la carrera de Lengua y Literatura Hispánicas de la Facultad de Educación e Idiomas de la Universidad Nacional Autónoma de Nicaragua, Managua, entre el 2009-2013. El objeto del estudio, a nivel general, es abordar la valoración y análisis que manifiestan los graduados y empleadores sobre tres grandes factores: la satisfacción, el desempeño laboral y el perfil profesional de los graduados. En este estudio, por considerarlo uno de los ejes vertebrales de la investigación, se ofrecen los resultados referidos al desempeño laboral de la población en estudio. La muestra está conformada por 174 graduados a quienes se les aplicó un cuestionario diseñado en escala Likert, utilizando categorías excluyentes, 
nominales e ítems de escala, en los que se les pide valoren su satisfacción ante su formación y su puesto de trabajo, su desempeño laboral y las características esenciales de su perfil profesional. El instrumento fue autoadministrado y respondido tanto por vía correo electrónico, como de manera directa. De acuerdo con los resultados obtenidos en el análisis de datos estadísticos mediante el programa SPSS v.21, un porcentaje muy alto (90\%) de los graduados encuestados se encuentra empleado en el área para la cual fueron formados (docencia en diferentes niveles de la educación), los graduados valoran muy positivo su desempeño laboral, no obstante, consideran que deben fortalecerse ciertas habilidades que les permitan desempeñar mejor su profesión.

\section{INTRODUCCIÓN}

La investigación que a continuación se presenta, surge ante el interés de conocer la problemática de los graduados de Lengua y Literatura Hispánicas del Departamento de Español de la Facultad de Educación e Idiomas de la UNAN-Managua ya que los estudios de la temática a nivel internacional Bedoya (2011), Campos (2010), Consejo Superior Universitario Centroamericano (CSUCA, (2004), Domínguez (2010), Justiniano (2006), Latorre (2010), entre otros, los ponen de relevancia como importante fuente de información para propósitos de mejoramiento institucional y en particular, un insumo fundamental para la autoevaluación institucional y para los procesos de acreditación, pues brindan a los responsables de las instituciones de educación superior, la oportunidad de analizar las características del desempeño profesional de sus egresados, con la posibilidad de contar con herramientas para reorientar los programas de estudio en términos de pertinencia ante la garantía de optar a mejores oportunidades en el mercado laboral de quienes se gradúan con dicha institución universitaria o de educación superior.

La temática de seguimiento a graduados es amplia, se caracterizará con detalle el desempeño laboral de los egresados de la carrera de Lengua y Literatura Hispánicas de la Facultad de Educación e Idiomas de la Universidad Nacional Autónoma de Nicaragua, Managua.

El interés para las universidades declina en ser organismos e instituciones eminentemente formadoras de profesionales que se insertan a un campo laboral específico, que a su vez, demanda de ellos, no solo la actualización constante de los conocimientos aprendidos, sino también la aplicación de competencias, destrezas y habilidades propias de dicho desempeño. El nivel competencial (conocimientos, aptitudes y habilidades...) deben ser evaluadas tanto por el empleador como por la universidad que los graduó, con el objetivo de verificar si los profesionales graduados responden a las necesidades de la sociedad y desempeñan eficientemente su función y de esta manera, comprobar la coherencia entre el perfil de egreso de los graduados y la práctica profesional de los mismos. Esperamos que esta investigación potencie un nuevo campo de estudio ante la comunidad científico-educativa y empresarial. 
Otro aspecto importante de este estudio, se relaciona con la entrada en vigencia de la Ley 704 (Ley creadora del Sistema Nacional para el aseguramiento de la calidad de la educación y reguladora del Consejo Nacional de Evaluación y Acreditación). La normativa permite a las universidades nicaragüenses prestar atención, con más ahínco de lo que tradicionalmente han venido haciendo, en el aseguramiento de calidad para satisfacer las necesidades, tanto de sus estudiantes como de la sociedad, respecto al mercado laboral, y una manera de medir la calidad de estas instituciones pasa por identificar el tipo de desempeño profesional de sus graduados en relación con la situación laboral y satisfacción ante el puesto de trabajo. Dicha ley, en su artículo 11, establece que las "Instituciones de Educación Superior, públicas y privadas legalmente establecidas en el país, desarrollarán procesos de autoevaluación institucional, a fin de identificar sus fortalezas y debilidades...".

Este razonamiento tiene un horizonte que sobrepasa las fronteras nacionales, siendo válido no solo para Nicaragua sino que, desde hace varios años se ha venido fortaleciendo a nivel internacional la tendencia de evaluación de la actividad universitaria, como una forma de rendición de cuentas a la sociedad y a los gobiernos. En ese contexto de evaluación, el seguimiento de egresados es un asunto de vital importancia para las universidades, ya que el desempeño profesional y personal de los egresados permite establecer indicadores con respecto a la calidad y eficiencia de las instituciones de educación superior (Asociación COLUMBUS, citado por Vega et. al. 2012, p. 31).

Estos procesos de acreditación están demandando la generación y exigiendo la realización de estudios de seguimiento de graduados por carrera específica, por tanto, las instituciones de educación superior requerirán de personal experto en el área de seguimiento a graduados para tener un referente de la calidad de la enseñanza brindada a sus graduados, en aspectos tales como los insumisos condiciones de funcionamiento, los procesos, los productos y resultados.

Dichas investigaciones además, permiten evaluar las necesidades de los egresados durante su práctica profesional y primer empleo para, de esta manera, mejorar su formación a través de la oferta permanente de formación de las universidades mediante la promoción de cursos de posgrado, maestrías, másteres y doctorados. Finalmente, se comparte con Lucio-Villegas y Cruz (1999), al ratificar en su estudio que desde esta perspectiva de acción formativa conjunta, se propicia la mejora en los procesos de comunicación entre la institución superior, los egresados y los empleadores.

Según la revisión de la lectura científica consultada y reseñada en líneas previas, los estudios de seguimiento a graduados, indudablemente han beneficiado a las instituciones de educación superior que los han realizado. Entre otros destacan: 
- Desarrollo de programas basados en las exigencias del contexto socioeconómico en el que se desenvolverán sus egresados, de manera que responden a las necesidades socioeconómicas actuales.

- Acceso al proceso de transición de la universidad al mercado laboral para valorar la calidad de dicha inserción y cuáles son los factores que favorecen o dificultan dicha inserción y así retroalimentar los procesos formativos.

- Evaluación de los planes formativos de los estudios superiores.

- Análisis y categorización de la realidad laboral de sus egresados, que a su vez, permitirá: Conocer información acerca del desempeño profesional, opiniones y sugerencias sobre la calidad de la educación recibida y de las nuevas demandas del mercado laboral y del medio social e; Identificar en qué medida se están alcanzando los fines de la educación y si los objetivos institucionales y curriculares se cumplen.

- Establecimiento de relaciones fluidas entre universidad y mercado laboral y mejora de la calidad en la oferta educativa para dotar al estudiante de mejores y mayores recursos al enfrentarse a la vida laboral y convertirse en profesionales agentes de cambio en la sociedad.

- Desarrollo de propuestas de evaluación de carácter continuo y sistemático de la institución universitaria con objeto de promocionar la innovación, impulsar nuevos modelos y métodos de enseñanza-aprendizaje y favorecer la optimización de recursos institucionales.

- Categorización y acuerdos que ayuden a unificar las perspectivas desde las cuales se realizan los estudios de seguimiento a graduados.

- Finalmente, mediante estos estudios se tiene acceso a un contenido estructurado sobre el proceso y resultado de la inversión social y económica que realiza el país al formar profesionales.

Como se puede apreciar, el estudio de seguimiento de egresados y su satisfacción laboral remite a destacar tres amplios ejes conceptuales y metodológicos enfocados, por un lado, en la gestión, organización y evaluación institucional de la propia universidad y, por otro, en las estructuras, características, desempeños laborales e intercambio comunicacional, empresauniversidad.

\section{MATERIAL Y MÉTODO}

Esta investigación se enmarca en un enfoque multimétodo, adscrita dentro del paradigma sociocrítico, pues no se adhiere solo al reduccionismo de datos de la corriente positivista (Cobos, 
2007) ni se aborda metodológicamente como una mera recopilación de datos para verificar teorías preconcebidas, pues como afirma Ricoy (2006), "se aspira desde el paradigma crítico a la unión entre la teoría y la práctica, usando la primera como fundamentación y guía de la segunda, con una interacción mutua" (p. 18).

Según la profundidad u objetivo, esta investigación es de tipo correlacional, de acuerdo con Hernández, Fernández y Baptista, los estudios correlacionales “... tienen como finalidad conocer la relación o grado de asociación que existe entre dos o más conceptos, categorías o variables en un contexto particular" (2010, p. 81). Se establecerán relaciones entre variables para identificar sentido de causalidad: significativa o no, rango de interacción entre las variables. Esta relación entre variables permitirá describir en mayor profundidad las características de la población en estudio.

Según el alcance temporal, este estudio es transversal (seccional o sincrónica) porque se estudiará a la población graduada de la carrera de Lengua y Literatura Hispánicas durante los años 2009-2013. Según Beltrán, del Rincón y Arnal, "este tipo de investigación estudia un aspecto de desarrollo de los sujetos en un momento dado" (2003, p. 44).

\subsection{Sujetos}

El universo del estudio está compuesto por todos los graduados entre el 2009-2013 de carrera de Lengua y Literatura Hispánicas de la Facultad de Educación e Idiomas de la UNANManagua que, según datos que lleva la Dirección de Registro Académico, Estadístico y Estudiantil, son 174 . En la figura 1 se presentan las características de la población en estudio.

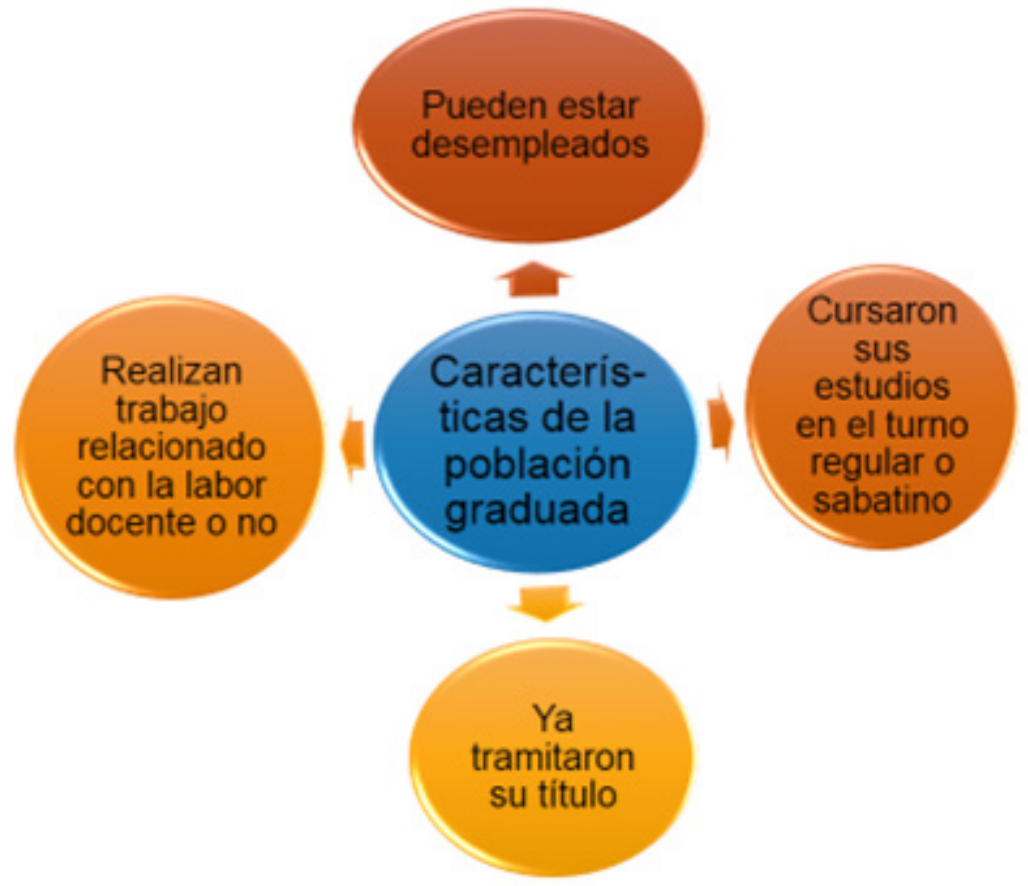

Figura 1: Características de la población en estudio. Elaboración propia. 
Se seleccionó una muestra probabilística estratificada (Hernández, Fernández y Baptista, 2010) por año de graduación (2009-2013), donde estuvieran representados los graduados de los diferentes años, de manera que fuera proporcional. Para tal fin, los datos fueron proporcionados por la Dirección de Registro Académico, Estadístico y Estudiantil y la Secretaría Académica de la Facultad de Educación e Idiomas.

Se considera que la muestra de este estudio es representativa de la población porque es un reflejo de ella, es decir, reúne las características principales de la población en relación con la variable en estudio. Además, los elementos de $\mathrm{N}$ se dividieron en varios estratos, de tal forma que, como afirma Ludewing (s.f.), cada estrato formado es internamente homogéneo porque sus integrantes se parecen mucho entre sí en lo que se refiere a la variable a estudiar, mientras que los estratos difieren unos de otros.

Como resultado de la aplicación de la fórmula estadística, la muestra del estudio quedó conformada por 120 graduados.

\section{$\mathbf{n}=\frac{\mathrm{Z}^{2^{*}} \mathrm{p}^{*} \mathrm{q}^{*} \mathrm{~N}}{\mathrm{~N}^{*} \mathrm{e}^{2}+\mathrm{Z}^{2^{*}} \mathrm{p}^{*} \mathrm{q}}$}

Donde: $Z^{2}=$ nivel de confianza (3.84 tabla de distribución normal para el $95 \%$ de confiabilidad); $\mathrm{N}=$ Población; $\mathrm{p}=$ probabilidad a favor (0.5); $\mathrm{q}=$ probabilidad en contra (0.5); $\mathrm{n}=$ tamaño de la muestra; e= margen de error 5\%; $\mathrm{N}=$ tamaño de la población.

\subsection{Instrumento}

En esta investigación se aplicaron, entre otras, técnicas de investigación documental y técnicas para la obtención de información en el campo, las cuales constituyeron la base de la fundamentación teórica y metodológica para el estudio. Con respecto a la primera, la revisión bibliográfica se realizó tanto en fuentes directas como indirectas, en físico, bases de datos, tesis, artículos de revista, como fuentes electrónicas situadas en la red Web.

En la obtención de información en el campo, se utilizó la técnica de la encuesta y como instrumento, se diseñó, pilotó e implementó un cuestionario. Se considera que las encuestas son una técnica adecuada ya que "proveen medios rápidos y económicos para determinar la realidad sobre los conocimientos, actitudes, creencias, expectativas y comportamientos de las personas (Beher, 2008, p. 62). Para garantizar que los datos obtenidos durante el proceso de recolección de información fueran de calidad, los instrumentos aplicados se redactaron de manera clara para que "todos los sujetos que respondan los entiendan de la misma manera. Para evitar ambigüedades en la redacción de los ítems, hay que tener en cuenta dos cuestiones 
fundamentales: Evitar expresiones negativas e incluir una sola idea en cada ítem (Behar, 2008, p. 78).

Para medir la actitud de los graduados frente a su situación laboral, se diseñó un cuestionario con preguntas cerradas (escala Likert), utilizando categorías excluyentes, nominales e ítems de escala, donde se les pide que valoren su grado de satisfacción y desempeño laboral, así como su formación profesional en la universidad.

Finalmente, en la elaboración del cuestionario, el primer paso fue el diseño la tabla de especificaciones o definición conceptual de las variables, cuya estructura se presenta en la tabla 1. En esta tabla se establecieron las dimensiones a partir de las cuales se definieron las categorías y subcategorías que permitieron la redacción de los ítems que en su conjunto dieran respuesta a cada uno de los objetivos específicos, lo cual se basó en la previa revisión bibliográfica.

Tabla 1. Tabla de especificaciones

\section{DIMENSIÓN: SATISFACCIÓN LABORAL}

Definición: Es el conjunto de actitudes generales del individuo hacia su trabajo.

\begin{tabular}{|c|c|c|c|c|c|c|}
\hline $\begin{array}{c}\text { Objetivos } \\
\text { Específicos }\end{array}$ & Categoría & Subcategoría & Ítems & Definición & Informante & Técnica \\
\hline
\end{tabular}

Una vez diseñado el cuestionario, se sometió a la valoración por parte de tres jueces, a quienes se les proporcionó el listado de ítems y una guía de observación para que presentaran sus puntos de vista y sugerencias en relación con su valoración sobre la calidad en la redacción de los ítems, su contenido y correspondencia con lo que se está investigando. Como resultado de este proceso, se hicieron ajustes al cuestionario, el cual, inicialmente, estaba compuesto por 51 preguntas cerradas y una abierta. Después de las diferentes revisiones por expertos se realizó un análisis, que llevó a reducirlo a 49 preguntas cerradas y una abierta.

Posterior a la valoración de los jueces se seleccionó una muestra de once participantes de la población en estudio para la aplicación piloto mediante correo electrónico. Producto de esta aplicación se agregó la escala NA (no aplica), por considerarse que no todos los encuestados trabajan en actividades relacionadas directamente con la docencia o bien, están en desempleo, por lo que no podían dar respuesta a algunas preguntas. En la tabla 2 se presenta la escala para cada dimensión del estudio.

Tabla 2. Escala final para cada dimensión de análisis

\begin{tabular}{|l|l|l|l|l|l|}
\hline \multicolumn{5}{|c|}{ DIMENSIÓN } & \multicolumn{5}{|c|}{ ESCALA } \\
\hline $\begin{array}{l}\text { Satisfacción } \\
\text { laboral }\end{array}$ & $\begin{array}{l}\text { Nada } \\
\text { Satisfecho }\end{array}$ & $\begin{array}{l}\text { Poco } \\
\text { satisfecho }\end{array}$ & No aplica & Satisfecho & $\begin{array}{l}\text { Muy } \\
\text { satisfecho }\end{array}$ \\
\hline
\end{tabular}




\begin{tabular}{|l|l|l|l|l|l|}
\hline DIMENSIÓN & \multicolumn{5}{|c|}{ ESCALA } \\
\hline $\begin{array}{l}\text { Desempeño } \\
\text { laboral }\end{array}$ & $\begin{array}{l}\text { Muy en } \\
\text { desacuerdo }\end{array}$ & $\begin{array}{l}\text { En } \\
\text { desacuerdo }\end{array}$ & No aplica & De acuerdo & $\begin{array}{l}\text { Muy de } \\
\text { acuerdo }\end{array}$ \\
\hline $\begin{array}{l}\text { Perfil } \\
\text { profesional }\end{array}$ & $\begin{array}{l}\text { Muy en } \\
\text { desacuerdo }\end{array}$ & $\begin{array}{l}\text { En } \\
\text { desacuerdo }\end{array}$ & No aplica & De acuerdo & $\begin{array}{l}\text { Muy de } \\
\text { acuerdo }\end{array}$ \\
\hline
\end{tabular}

El siguiente paso consistió en medir la confiabilidad del cuestionario para lo que se aplicó la fórmula de Alpha de Cronbach, porque, como afirma Corral (2009), es utilizada para:

Evaluar la confiabilidad o la homogeneidad de las preguntas o ítems [...] cuando se trata de alternativas de respuestas policotómicas, como las escalas tipo Likert; la cual puede tomar valores entre 0 y 1 , donde: 0 significa confiabilidad nula y 1 representa confiabilidad total (p. 241).

Los resultados de este proceso indican que el cuestionario es altamente confiable ya que el resultado es mayor a .60 .

En la tabla 3 se muestran los resultados.

Tabla 3. Estadísticos de fiabilidad para cada dimensión del estudio

\begin{tabular}{|c|c|c|}
\hline DIMENSIÓN & ALFA DE CRONBACH & N DE ELEMENTOS \\
\hline Satisfacción laboral & .831 & 16 \\
\hline Desempeño laboral & .935 & 9 \\
\hline Satisfacción laboral & .818 & 11 \\
\hline
\end{tabular}

La decisión de diseñar un cuestionario con estructura de pregunta cerrada se debe a que presenta múltiples ventajas:

- Requiere de un menor esfuerzo por parte de los encuestados

- Limitan las respuestas de la muestra

- Es fácil de llenar

- Es relativamente objetivo

- Es fácil de clasificar y analizar (Behar, 2008, p. 65)

\subsection{Procedimientos}

Para proceder a la aplicación de la encuesta, se informó vía telefónica a los graduados sobre el estudio que se estaba realizando y se les pidió su colaboración en el cumplimentado o 
llenado de la encuesta. A los que contaban con una dirección de correo se les envió la encuesta y a los que no, se les hizo una visita a su domicilio.

Una vez recogidos los cuestionarios, se procedió al vaciado de los datos y su posterior análisis y discusión de los resultados con la ayuda de SPSS V 21, mediante el cual se calcularon estadísticos descriptivos como frecuencias absolutas de cada categoría para descartar la presencia de valores ajenos a los valores originales de cada variable. Seguidamente, se procedió al análisis de datos con la elaboración de gráficos, que permitieron realizar un análisis descriptivo de la muestra, atendiendo a los diferentes apartados en que se encuentra estructurado el cuestionario (satisfacción, desempeño laboral y perfil profesional).

\section{RESULTADOS}

En este artículo se presentan los resultados correspondientes a la dimensión de estudio: desempeño laboral, que permitió conocer cómo valoran los graduados su desempeño laboral. Se aborda este apartado de la investigación, por considerarse que la formación profesional del individuo repercute en su desempeño laboral y en la medida en que su formación sea sólida, así será su desempeño, razón por la que las instituciones formadoras de profesionales toman como referente de mejora los resultados de estudios de seguimiento a graduados, porque les va a permitir formar profesionales competentes para las instituciones que emplean a los graduados, pues el desempeño laboral es la base fundamental para el desarrollo de cualquier institución. Para Palaci (citado por Pedraza, Amaya y Conde, 2010):

El desempeño laboral es el valor que se espera aportar a la organización de los diferentes episodios conductuales que un individuo lleva acabo en un período de tiempo. Estas conductas, de un mismo o varios individuo (s) en diferentes momentos temporales a la vez, contribuirán a la eficiencia organizacional (p. 493).

A continuación se presentan los resultados correspondientes a los 9 ítems orientados a medir el desempeño laboral de los encuestados.

\section{Ítem 1: Selecciono, elaboro y utilizo materiales didácticos pertinentes con el contexto social y político de los estudiantes}

Una selección adecuada de los materiales didácticos conlleva a un eficiente proceso de aprendizaje por parte del discente. Según se aprecia en la figura 2, el 89 \% de los encuestados manifiestan estar de acuerdo y muy de acuerdo en que seleccionan, elaboran y utilizan materiales didácticos pertinentes con el contexto social y político de los estudiantes, un porcentaje bastante alto. Solo un $1 \%$ está en desacuerdo y el $10 \%$ no aplica, este último porcentaje corresponde a los encuestados que no trabajan directamente en la docencia o están desempleados. 


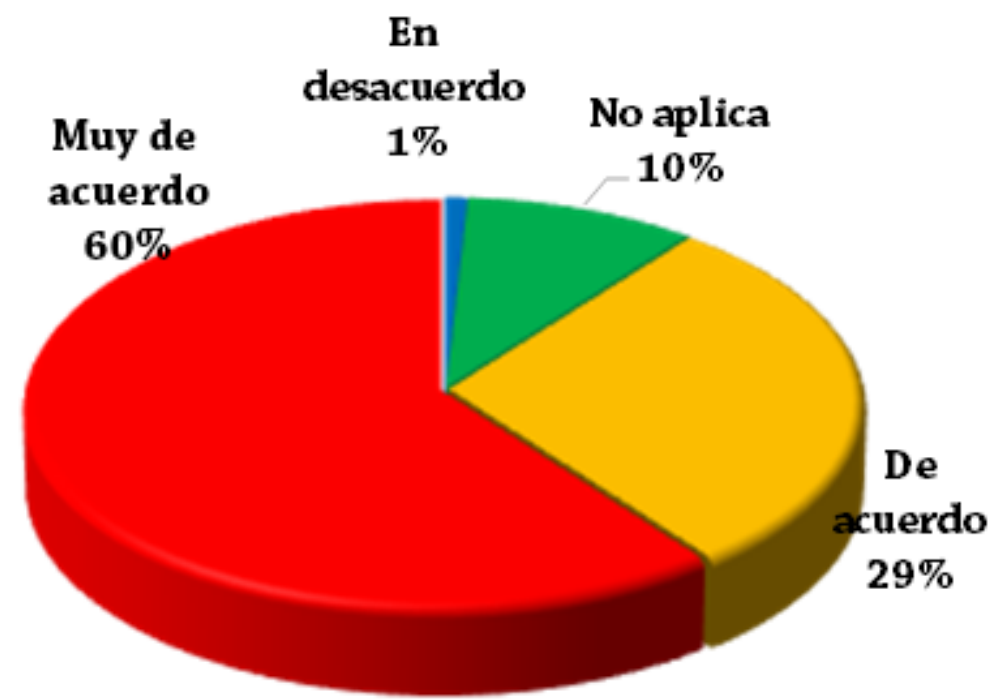

Figura 2. Pertinencia de los materiales didácticos. Elaboración propia

\section{Ítem 2: Los materiales didácticos que selecciono y elaboro favorecen los procesos de enseñanza-aprendizaje}

Los materiales didácticos son fundamentales en el proceso de enseñanza-aprendizaje, pues en la medida en que el discente se identifique con ellos y logre su apropiación, se facilitará su comprensión y aprehensión. Los resultados ante la pregunta sobre si los materiales didácticos que seleccionan y elaboran, los encuestados favorecen los procesos de enseñanza-aprendizaje, son exactamente igual que la pregunta anterior: 89 \% están de acuerdo, un $1 \%$ en desacuerdo y un $10 \%$ no aplica, lo que se puede ver en la figura 3.

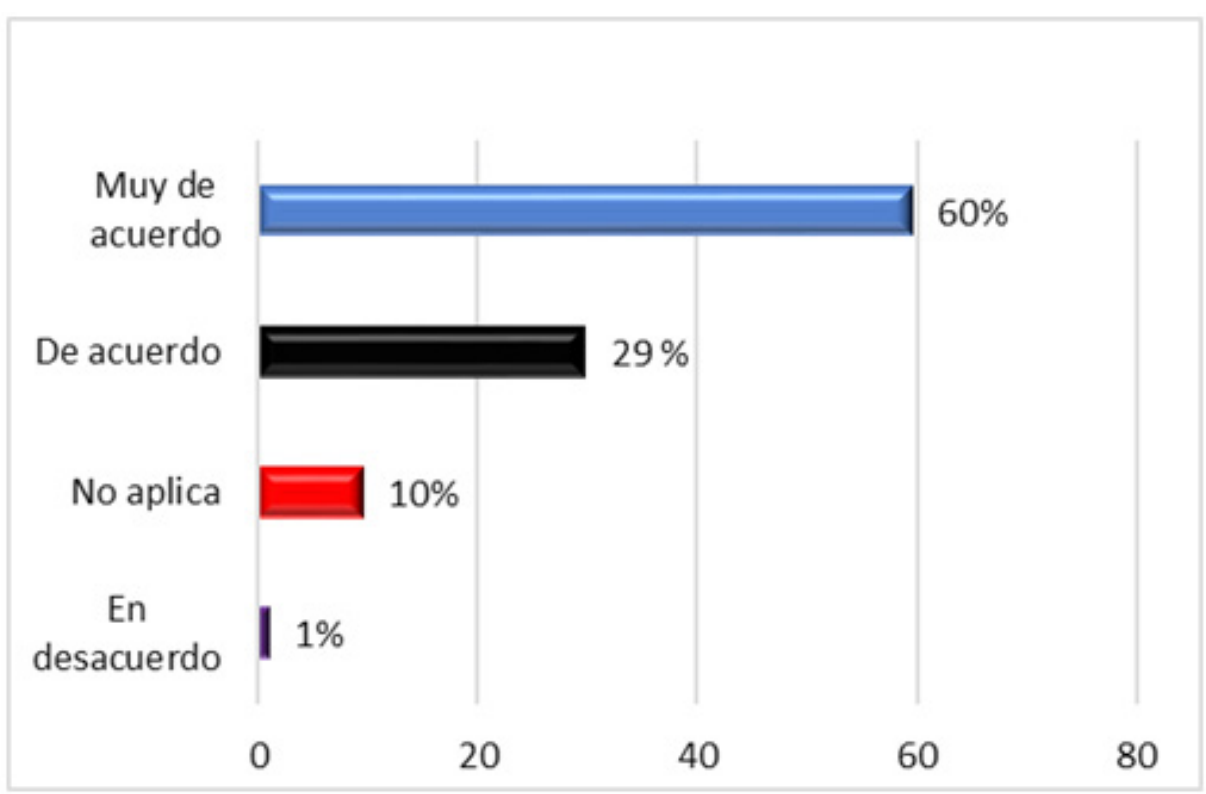

Figura 3. Los materiales seleccionados favorecen el proceso de enseñanza aprendizaje. Elaboración propia 


\section{Ítem 3: Estoy pendiente del progreso y rendimiento académico de los discentes}

Una de las funciones del docente es dar seguimiento al rendimiento del estudiantado a su cargo para garantizar un óptimo rendimiento académico. En la figura 4 se puede ver que el 68 $\%$ de los encuestados están muy de acuerdo en que están pendientes del progreso y rendimiento académico de sus discentes $21 \%$ está de acuerdo, $9 \%$ no aplica y $2 \%$ no responde. En el caso del porcentaje que respondió que no aplica, está relacionado con el porcentaje desempleado y con los que no están relacionados directamente con la docencia.

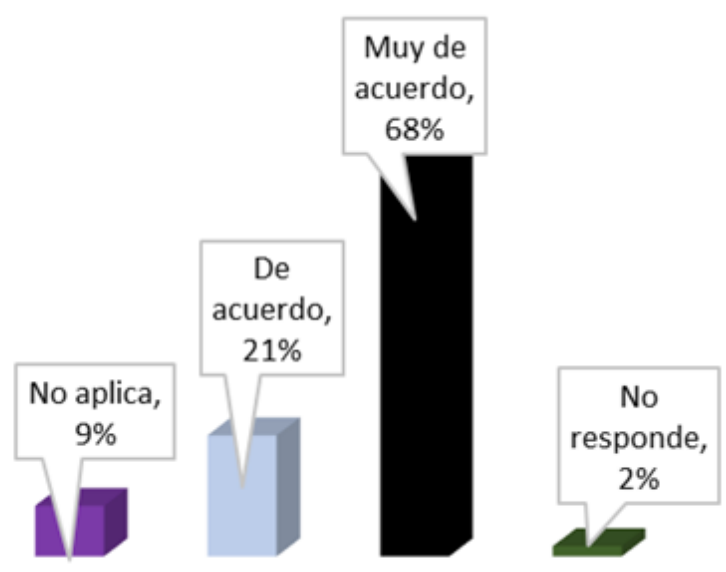

Figura 4. Estoy pendiente del progreso de mis alumnos. Elaboración propia.

\section{Ítem 4: Aplico técnicas adecuadas para avaluar los conocimientos de mis alumnos}

Las evaluaciones en el proceso enseñanza-aprendizaje son relevantes porque permiten medir el grado de conocimientos y competencias adquiridas por los discentes. En la figura 5 se aprecia que el $62 \%$ y $28 \%$ de los encuestados están muy de acuerdo y de acuerdo, respectivamente, en que aplican técnicas adecuadas para evaluar los conocimientos de sus alumnos, el restante $10 \%$ responde que no aplica.

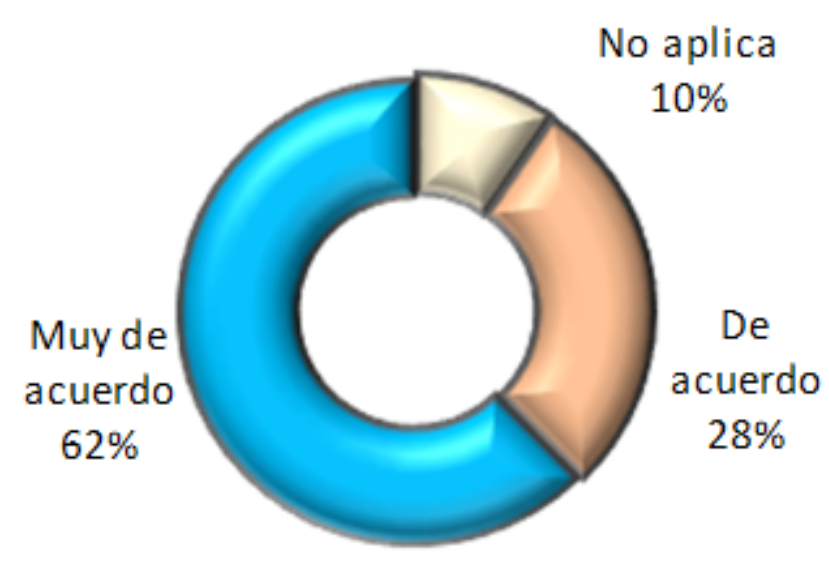

Figura 5. Técnicas adecuadas de evaluación Elaboración propia 


\section{Ítem 5: Contribuyo al fortalecimiento de los valores éticos de mis discentes}

La formación de un individuo va más allá de la adquisición de conocimientos científicos, gran parte del desarrollo de las competencias deseables las desarrollará en la medida en que sus valores éticos se fortalezcan. En la figura 6 se aprecia que el $77 \%$ de los encuestados está muy de acuerdo en que contribuye al fortalecimiento de los valores éticos de los alumnos, el $14 \%$ está de acuerdo y el $9 \%$ no aplica.

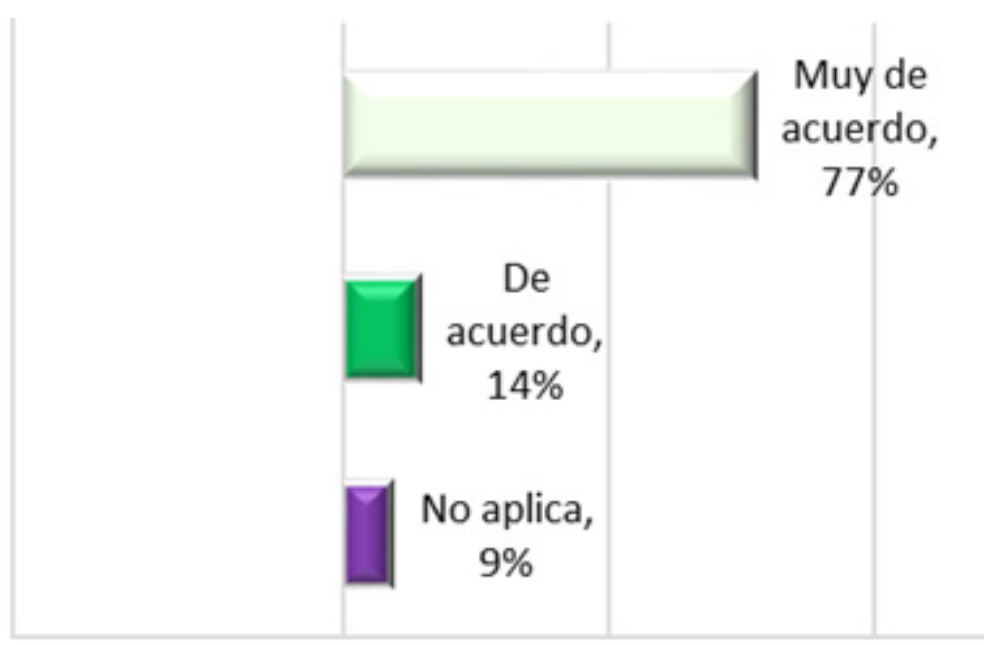

Figura 6. Formación de valores éticos. Elaboración propia.

\section{Ítem 6: Soy capaz de resolver situaciones problemáticas de mi área de trabajo}

Todo trabajador cada día se enfrenta a situaciones problemáticas a las cuales debe dar solución de manera efectiva para que su desempeño sea eficiente. De acuerdo con la figura gráfico 7, el $96 \%$ de los encuestados están muy de acuerdo y de acuerdo (60\% y 36\%) en que son capaces de resolver problemas de su área de trabajo, independientemente de que no esté relacionado con el área docente. El restante $4 \%$ responde que no aplica, coincide con el segmento que se encuentra en desempleo.

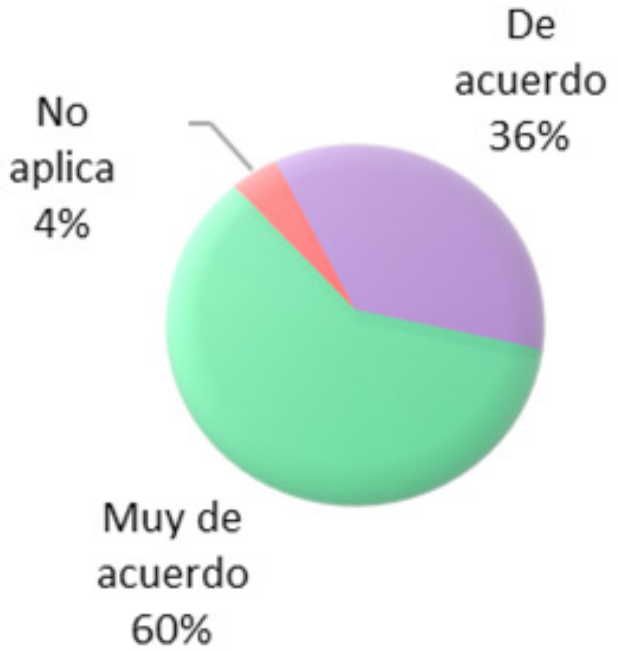

Figura 7. Resuelve situaciones problemáticas en su área de trabajo. Elaboración propia. 


\section{Ítem 7: Constantemente estoy innovando los métodos de trabajo con los discentes}

La innovación en los métodos de enseñanza se considera fundamental en una sociedad donde los cambios en todas las áreas son vertiginosos, y la educación no puede quedarse rezagada. Según se observa en la figura 8, la opinión de los encuestados, respecto a si constantemente innovan los métodos de trabajo con los estudiantes, está dividida en muy de acuerdo y de acuerdo, con $46 \%$ cada una. El $8 \%$ restante no aplica; un porcentaje, por encontrarse en desempleo y otro, por no trabajar directamente en el aula de clase.

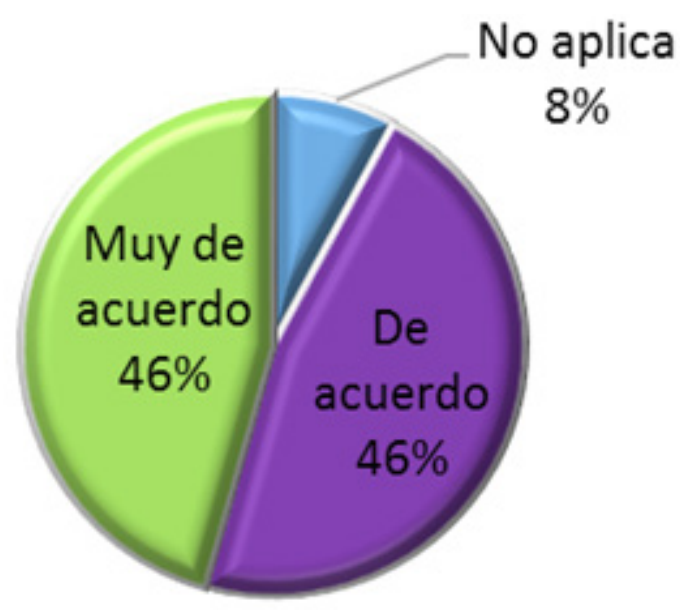

Figura 8. Innovación en los métodos de trabajo. Elaboración propia.

\section{Ítem 8: Soy disciplinado y organizado en mi trabajo}

No solo las competencias y conocimientos científicos, técnicos y metodológicos garantizan un buen desempeño profesional, son importantes además, aquellos valores y actitudes que contribuyen a la aplicación eficaz de los primeros. En la figura 9 se puede ver que del total de encuestados que están empleados, $96 \%$, consideran que son disciplinados y organizados en su trabajo (68\% muy de acuerdo y $28 \%$ de acuerdo).

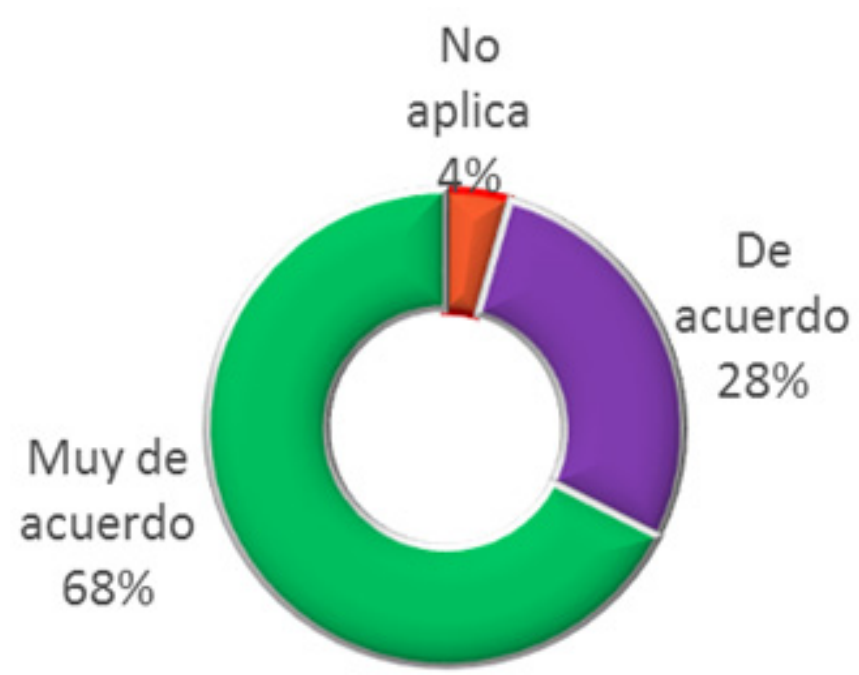

Figura 9. Disciplina y organización en su trabajo. Elaboración propia. 


\section{Ítem 9: Cumplo con responsabilidad mis deberes laborales}

También es favorable la opinión respecto a que los encuestados cumplen con responsabilidad sus deberes laborales. En la figura 10 se observa que 76 \% está muy de acuerdo y $20 \%$ de acuerdo.

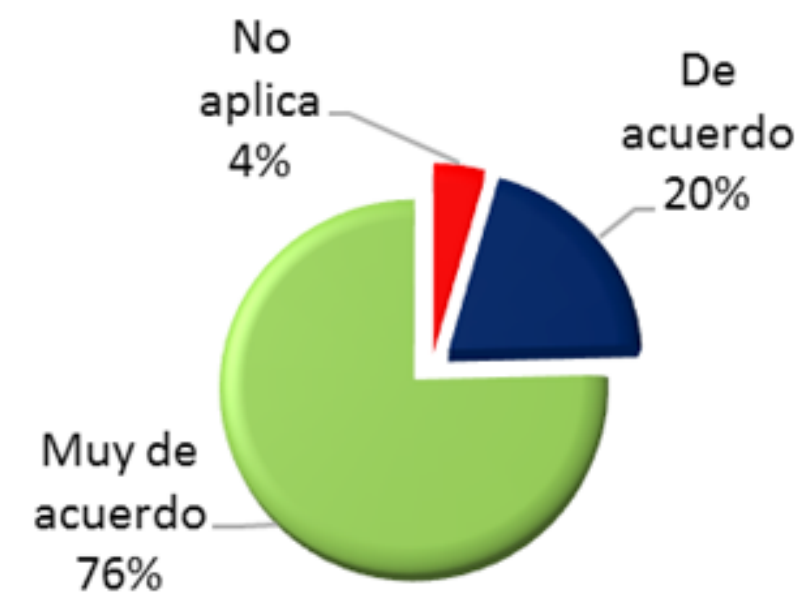

Figura 10. Responsabilidad en el trabajo. Elaboración propia.

\section{CONCLUSIONES}

En las conclusiones extraídas a la luz de los resultados obtenidos, se plasman los aspectos más relevantes que podrían ser de utilidad para las Instituciones de Educación Superior donde se imparte la carrera de Lengua y Literatura Hispánicas. Se intentará, a través de las mismas, dar respuesta al problema de investigación planteado, sobre el desempeño laboral de los graduados de Lengua y Literatura Hispánicas del Departamento de Español de la Facultad de Educación e Idiomas de la UNAN-Managua. La relevancia de esta investigación radica en que es el primer estudio sobre seguimiento a graduados que se realiza en Nicaragua y por tanto, las conclusiones contribuirán a mejorar aquellos aspectos que son relevantes en la formación profesional del graduado al momento de ejercer su profesión.

La dimensión desempeño laboral fue valorada tomando en cuenta destrezas, habilidades, actitudes y aptitudes que el graduado debe poner en práctica para realizar adecuadamente su trabajo. La gran mayoría de los graduados (92 \%), tiene una apreciación favorable al respecto. De acuerdo con la encuesta aplicada, se consultó sobre conocimientos académicos específicos de la carrera y actitudes, aspectos que están relacionados con los factores individuales de la empleabilidad (Oliván, s.f.). Por parte de los empleadores, se mencionó que el desempeño laboral de los graduados se debe a factores también internos de la empleabilidad: empleo de redes sociales, metodología, planificación, puntualidad, optimismo, relaciones personales. Estas habilidades se identifican en los graduados y se corresponden con las competencias sistémicas valoradas en importancia y desarrollo por los estudiantes españoles de las Universidades de Huelva y la Complutense de Madrid (Alonso, Cruz y Hermosín, 2010). 
Sobre la base de los resultados encontrados, esta investigación permitirá al Departamento de Español de la Facultad de Educación e Idiomas de la UNAN-Managua:

- Conocer información acerca del desempeño profesional, opiniones y sugerencias acerca de la calidad de la educación recibida y de las nuevas demandas del mercado laboral y del medio social.

- Analizar en qué medida se están alcanzando los fines de la educación y si los objetivos institucionales y curriculares se cumplen.

- Conocer la realidad laboral de sus egresados.

- Promover entre los graduados de Lengua y Literatura Hispánicas los programas de posgrado que se ofrecen en este Departamento Docente, de modo que puedan especializarse en un área y actualizar los conocimientos adquiridos durante la carrera.

\section{REFERENCIAS BIBLIOGRÁFICAS}

Alonso, P., Cruz, M. R. y Hermosín, M. J. (2010). Análisis del Nivel de desarrollo e Importancia de las Competencias transversales en el alumnado de la Facultad de Ciencias la Educación para la mejora de la calidad Docente. Barcelona.

Bedoya, C. (2011). Los imaginarios sociales de los egresados de pregrado de la Universidad Cooperativa de Colombia sede Cartago sobre la calidad de la formación (Tesis de maestría, Universidad Autónoma de Manizales, Colombia). Recuperado de: http:// bibliotecavirtual.clacso.org.ar/Colombia/ alianzacindeumz/20130319010509/ TesisClaudiaLilianaBedoyaAbella.pdf

Behar, D. (2008). Metodología delaInvestigación. Recuperado de: http://yoprofesor. org/2016/03/23/metodologia-de-lainvestigacion-por-behar-rivero-en-pdf/

Beltrán, A., Del Rincón, D. y Arnal, J. (2003). Bases metodológicas de la investigación
Educativa. Barcelona, España. Ediciones Experiencia, S.A.

Campos, M. (2010). Estudio sobre la inserción laboral de los egresados de Magisterio de Educación Física de la Universidad de Sevilla (Tesis doctoral). Universidad de Sevilla, España. Recuperado de: http://fondosdigitales.us.es/media/ thesis/1347/K_Tesis-PROV28.pdf/

Cobos, D. (2007). Reflexiones sobre la investigación educativa. Escuela Abierta, (10), 213-221. Recuperado de: file:///C:/ Users/Pc/Downloads/Dialnet-Refle xionesSobreLaInvestigacionEducati va-2520042\%20(1).pdf

Consejo Superior Universitario Centroamericano. (2004). Los estudios de seguimiento a egresados en América Central: un esfuerzo para mejorar la relevancia de los programas de estudio en las universidades. Recuperado de: http://daad.CSUCA. 
org/attachments/121_MEMORIA\%20

E S TUDIO \% 20 S E G U I M I E N T O 20 DE\%20EGRESADOS.pdf

Domínguez, R. (2010). Desempeño y satisfacción de los graduados de un programa de formación docente (Tesis de Maestría Universidad Autónoma de Yucatán). Recuperado de: http://cybertesis.unmsm. edu.pe/bitstream/cybertesis/3981/1/ Tolentino_sl.pdf/

Hernández, R., Fernández, C. y Baptista, P. (2010). Metodología de la Investigación. México. Mc Graw Hill.

Justiniano, M. (2006). La inserción laboral de los profesionales graduados en ciencias de la educación (Tesis doctoral, Universidad de Barcelona, España). Recuperado de: http://diposit.ub.edu/dspace/ bitstream/2445/42458/1/MDJ_TESIS. pdf

Latorre, I. (2010). Inserción socio profesional de egresados de bachilleratos y posgrados musicales en instituciones de enseñanza superior de Puerto Rico (Tesis doctoral) Universidad de Granada, España. Recuperado de: http://digibug.ugr.es/ bitstream/10481/6623/1/18936106.pdf/

Ley Creadora del Sistema Nacional para el aseguramiento de la calidad de la educación y reguladora del Consejo Nacional de Evaluación y Acreditación. La Gaceta $N^{\circ} 172$. Managua, Nicaragua, 12 de septiembre de 2011.
Lucio-Villegas, E y Cruz, M. R. (1999). Algunas aportaciones a temática de la formación en la empresa. Bordón. Revista de Pedagogía 51(1), 51-59.

Ludewing, C. (s.f.). Universo y Muestra. Recuperado de: http://www.smo.edu.mx/ colegiados/apoyos/muestreo.pdf/

Oliván, C. (s.f.). Empleabilidad y Empleo: formación universitaria y mercado de trabajo en España y Aragón. Recuperado de: $\quad$ www.aragon.es/.../140511\%20 Empleabilidad\%20y\%20Empleo.pdf/

Pedraza, E., Amaya, G. y Conde, M. (2010). Desempeño laboral y estabilidad del personal administrativo contratado de la Facultad de Medicina de la Universidad del Zulia. Revista de Ciencias Sociales. 16(3), 493-505.

Ricoy, C. (2006). Contribución sobre los paradigmas de investigación. Revista do Centro de Educação, XXI(1), 11-22. Recuperado de: http://www.redalyc.org/ pdf/1171/117117257002.pdf/

Vega, A., González, E., Toro, G., Fonseca, G., Sánchez, J., Peña, M. y Carrasco, S. (2012). Aspectos referenciales sobre la inserción laboral y seguimiento de egresados. En Centro Interuniversitario de Desarrollo (CINDA) (Ed.). Santiago de Chile: Alfabeta Artes Gráficas. Recuperado de: http:// www.cinda.cl/.../libros/01\%20Cinda\%20 Seguimiento\%20indd\%20(2).pdf/ 